\title{
OPTIMUM LINEAR SYSTEMS FOR POWER CONSTRAINED TRANSMISSION
}

\author{
Israfil Bahceci and Amir K. Khandani \\ University of Waterloo \\ Electrical and Computer Engineering Department \\ Waterloo, ON N2L3G1 Canada
}

\begin{abstract}
This paper studies transmission of sources over noisy channels by a communication system subject to a power constraint at the transmitter. We consider the transmission of continuous- or discrete-time Gaussian sources and assume that the processing on the signals are within the family of linear operations. For stationary signals, a constraint on the power at the output of the transmission filter results in bandpass transmit and receive filters whose bandpass region include the frequencies at which the source spectra is above some threshold. This result shows similarities between the rate distortion arguments and the minimum mean square error (MSE) attainable by the optimum linear systems. Examples for frequently encountered source statistics are also studied and it is shown that linear processing both at the transmitter and at the receiver reduces the estimation errors significantly compared to the standard Wiener filtering approach. It is shown that for communication of a bandlimited source with arbitrary spectrum over a bandlimited channel with the same banswidth as the source, using proposed linear processing approach attains the same distortion exponent as the theoretically optimum system does.
\end{abstract}

\section{INTRODUCTION}

Consider the transmission of an analog Gaussian source over a noisy channel where the aim is to attain as small distortion as possible between the source and its decoded version. It is known that if the source is Gaussian emitting independently and identically distributed samples and the channel is an additive white Gaussian noise (AWGN) channel being used only once per source sample, the optimum system minimizing the square error distortion is achieved by plugging the source output to the channel by an amplifier and recovering it by attenuating the signal at the channel output $[1,2]$. This sort of uncoded transmission is optimal also for an ideal bandlimited continuous Gaussian source being transmitted over a bandlimited channel with the same bandwidth as the source. While it is attractive to realize optimum systems with such simplicity, in general, it might be necessary to resort to more complex encoding/decoding architectures to attain the optimum system performance $[2,3]$.

Two critical constraints in a communication system are (i) time delay of coding/decoding, and (ii) transmission power/energy. In many systems, such as sensor networks, these two constraints play a fundamental role in the design. When the bandwidth is scarce, the source contains delay sensitive information and the transmitter has a small power source, it is not viable to wait for a long time to collect data and apply very long source/channel codes (generally consisting of a suitable quantizer followed by a suitable channel code) and highly complex decoding schemes [2]. It might be more useful to obtain timely information at the receiver even if the resulting accuracy may not be at the optimum level.
In this paper, we address the point-to-point communication of a source under the two constraints mentioned above. First, we restrict the encoding and decoding processes to be in the class of linear operations (See Figure 1). Thus, the source stream is plugged to the channel through just a transmit filter, and then at the receiver, it is reconstructed from the noisy version of the transmitted signal using another filter. Communication via linear systems has been treated in the literature [4-10]. In [5-7], optimum transmitter and receiver design is studied considering pulse amplitude modulation. The transmission of discrete time memoryless vector source over a vector channel are considered in [8-10]. Costas [4] studies message transmission with linear pre- and post-filters, however, his work lacks an information theoretic basis. Our motivation in this paper is to develop optimum linear processing schemes for the transmission of a more general class of analog or discrete sources over an arbitrary linear filter channel. We cast the optimum encoding/decoding problem as a linear filter design problem subject to a power constraint at the output of the transmitter. For stationary sources, the closed form expressions for the frequency responses of the optimal filters indicate that an optimal transmit filter is a bandpass filter which passes the frequencies at which the power spectrum of the signal normalized by the noise spectrum is above some threshold. This is consistent with the optimum rate-distortion criterion which states that an efficient representation of the source should ignore the frequency components at which the power spectrum density is small [2, 3]. Similar results apply to the discrete-time case as well but due to the limited space, they are omitted here.

The organization of the paper is as follows: In the next section, we introduce the system model being considered and develop the formulation for the optimal filter design. In Section 3, the optimum filters that minimize the mean square error (MSE) are derived. In this section, we also consider the theoretically optimum system performance through rate-distorion function, and provide numerical examples that support the use of optimal transmit and receive filters. Finally, in Secton 4, we provide concluding remarks.

\section{SYSTEM MODEL}

A general linear time invariant communication system is depicted in Figure 1. This figure assumes continuous-time signals and systems, however, the case for discrete-time follows similarly and parallel results can be obtained; therefore, our focus is on continuous-time systems for the time being. The boxes $A$ and $B$ denote the linear transmit and receive filters whose impulse responses at time $\tau$ to an impulse at time 0 are denoted by $A(\tau)$ and $B(\tau)$, respectively. The channel transfer matrix is denoted by $H(\tau)$. For the stationary source and a time-invariant channel, the filters can be represented in terms of frequency responses, $A(w), H(w)$, and $B(w)$. The re- 


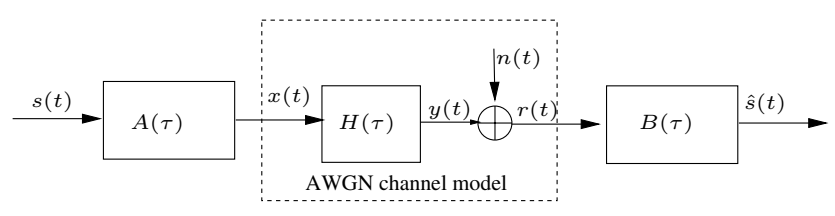

Fig. 1. Block diagram for a linear communication system

sponse of the transmit filter, $x(t)$, to a random signal, $s(t)$ (which is assumed to be output of a Gaussian source), can be expressed as $x(t)=\int s(\tau) A(t-\tau) d \tau$. The signal at the receiver, $r(t)$, is a corrupted version of $x(t)$ by the channel $H(t, \tau)$ and the additive Gaussian noise (AWGN), $n(t): r(t)=\int x(\tau) H(t-\tau) d \tau+n(t)$, and passed through the filter $B$ to obtain the estimate $\hat{s}(t)$ for $s(t)$, $\hat{s}(t)=\int r(\tau) B(t-\tau) d \tau$. The objective is to find the filters that minimize the MSE $\xi=E\left\{|s(t)-\hat{s}(t)|^{2}\right\}$, subject to the power constraint $E\left\{|x(t)|^{2}\right\} \leq P$.

If we remove the transmit filter and the power constraint (e.g., assuming $A$ is the identity operation), this problem reduces to the signal estimation problem [11]. For stationary sources, the solution is given by a Wiener filter [12]. For non-stationary sources, the timevarying filter response should satisfy an integral equation [13]. As we will see later, a linear mapping at the transmitter side will be useful since the transmitted signal can be optimized according to the channel characteristics and the bandwidth and power requirements.

\section{OPTIMUM LINEAR PROCESSING FOR CONTINUOUS TIME SIGNALS}

\subsection{Optimum Linear Filters}

For a wide-sense stationary signal $s(t)$ with power spectral density $S(w)$, the filtering processes by the the transmit, channel and receive filters reduce to the well-known convolution integrals and we have the power spectral density expressions for $x(t)$ and $\hat{s}(t)$ as $S_{x}(w)=|A(w)|^{2} S(w)$, and $\hat{S}(w)=S_{r}(w)|B(w)|^{2}$, respectively, where $S_{r}(w)=S_{x}(w)|H(w)|^{2}+S_{n}(w)$. Hence, the optimization problem for $A(w)$ and $B(w)$ can now be cast as

$$
\begin{array}{cc}
\min & \frac{1}{2 \pi} \int S(w) \mid\left(1-\left.A(w) H(w) B(w)\right|^{2} d w+\frac{1}{2 \pi} \int S_{n}(w)|B(w)|^{2} d w\right. \\
\text { s.t. } & \frac{1}{2 \pi} \int S(w)|A(w)|^{2} d w \leq P .
\end{array}
$$

Solving for $A(w)$ and $B(w)$ by Lagrange multiplier method (for brevity, we omit the details), we can obtain

$|A(w)|^{2}=\left\{\begin{array}{cc}\frac{S_{n}(w)}{S(w)|H(w)|^{2}}\left[\sqrt{\frac{S(w)|H(w)|^{2}}{\lambda S_{n}(w)}}-1\right], & \frac{S(w)|H(w)|^{2}}{S_{n}(w)} \geq \lambda \\ 0, & \text { else }\end{array}\right.$

and

$$
B(w)=\left\{\begin{array}{cc}
\sqrt{\frac{\lambda S(w)}{S_{n}(w)|H(w)|^{2}}} A^{*}(w) H^{*}(w), & \frac{S(w)|H(w)|^{2}}{S_{n}(w)} \geq \lambda \\
0, & \text { else }
\end{array}\right.
$$

where $\lambda$ satisfies

$$
\sqrt{\lambda}=\frac{\int_{\Omega} \sqrt{\frac{S_{n}(w) S(w)}{|H(w)|^{2}}} d w}{2 \pi P+\int_{\Omega} \frac{S_{n}(w)}{|H(w)|^{2}} d w}
$$

and $\Omega=\left\{w: S(w)|H(w)|^{2} \geq \lambda S_{n}(w)\right\}$. A graphical representation to determine the optimal filter is depicted in Figure 2. The optimal transmission filter $A$ passes the frequencies at which the source spectral density normalized by the noise power are above some threshold $\lambda$ which is determined by (4). The minimum mean square with the optimum filters is given by

$$
\xi_{i, o}=\frac{1}{2 \pi} \int_{\Omega^{c}} S(w) d w+\frac{\sqrt{\lambda}}{2 \pi} \int_{\Omega} \sqrt{\frac{S_{n}(w) S(w)}{|H(w)|^{2}}} d w
$$

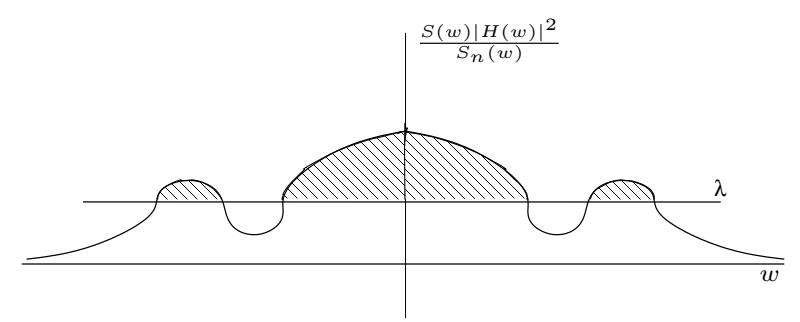

Fig. 2. The frequency of the optimal filters are nonzero for $\frac{S(w)|H(w)|^{2}}{S_{n}(w)}>\lambda$

The optimum transmission filter defined by (2) can be imagined as a cascade of two filters as depicted in Figure 3.a. The response of the first filter is $1 / T^{+}(w)$ which is obtained by factoring out $T(w)=\frac{S(w)|H(w)|^{2}}{S_{n}(w)}$ such that $T^{+}(w)$ has the left-half plane poles and zeros of $T(w)$. Hence, the first filter is realizable. On the other hand, the response of the second filter is given by

$T_{2}(w)=\left\{\left[\sqrt{\frac{S(w)|H(w)|^{2}}{\lambda S_{n}(w)}}-1\right]^{1 / 2}, \quad \frac{S(w)|H(w)|^{2}}{S_{n}(w)} \geq \lambda\right.$,

which is not realizable since it does not satisfy the Paley-Wiener criterion. However, one may approximate this filter with a realizable one. A similar design method can also be pursued for the optimum receive filter, $B(w)$ (See Figure 3.b.). From (3), we observe that $B(w)$ can be viewed as a cascade of three filters: (i) a standard wiener filter for the filtering problem $r(t)=y(t)+n(t)$, $T_{i}(w)=\frac{S_{y}(w)}{S_{r}(w)}$, (ii) the reciprocal of $A(w), T_{i i}(w)=1 / A(w)$, and (1) (iii) the channel inversion filter, $T_{i i i}(w)=1 / H(w)$.

\subsection{Theoretically Optimum Performance}

The optimal performance of the above communication system can be evaluated by the rate-distortion bounds and the channel capacity expressions [2,3]. The rate-distortion function, $R(D)$, provides the minimum rate required to attain some distoriton level $D$, whereas the capacity function, $C(\rho)$, gives the maximum information rate that can be transferred across a noisy channel at a signal-to-noise ratio of $\rho$. Hence, we have $R(D) \leq C(\rho)$, and the theoretical minimum distortion $D_{\min }$ satisfies $R\left(D_{\min }\right)=C(\rho)$. Hence, we can obtain the optimal performance of the system in Figure 1. To illustrate the relation between the performance of optimal linear filtering and the theoretical optimal system, let us consider the infinite bandwidth AWGN channel with $S_{n}(w)=N_{0} / 2$. The theoretical minimum mean square error distortion is obtained by solving

$$
R(D)=\frac{P}{N_{0}}=\frac{1}{2 \pi} \int_{S(w)>\frac{1}{2 v}} \frac{1}{2} \log (2 v S(w)) d w
$$



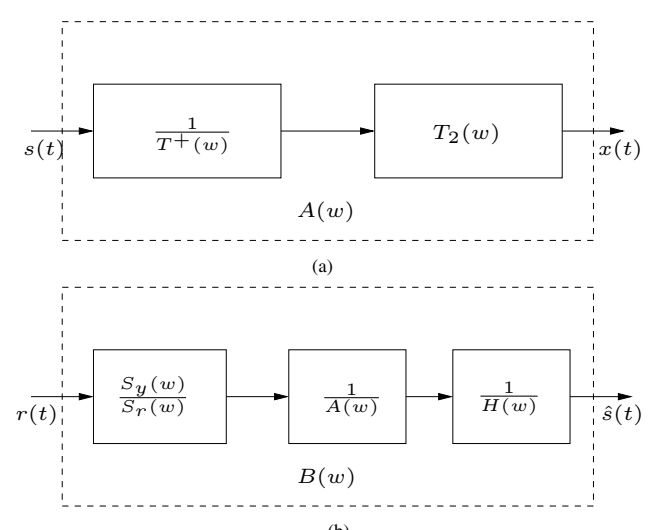

(b)

Fig. 3. Realization of optimum filters as a cascade of subfilters, (a) transmit filter $(b)$ receive filter

for $v$, and substituting the solution to

$$
D=\frac{1}{2 \pi} \int_{S(w) \leq \frac{1}{2 v}} S(w) d w+\frac{1}{2 v 2 \pi} \int_{S(w)>\frac{1}{2 v}} d w
$$

After some manipulations, we can rewrite (7) as

$\frac{P}{N_{0}}+\left(\log \frac{1}{2 v}\right) \frac{1}{2 \pi} \int_{S(w)>\frac{1}{2 v}} d w=\frac{1}{2 \pi} \int_{S(w)>\frac{1}{2 v}} \log S(w) d w$.

Note that the Equalities (9) and (4) that $\nu$ should satisfy for the case of optimum theoretical system and the optimal linear systems have very similar forms (Set $S_{n}(w)=N_{0} / 2$ and $H(w)=1$ in (4)). A similar correspondence exists also between the theoretical minimum MSE given by (8) and the minimum MSE attained by the optimal linear filtering given in (5). While an immediate insight is not possible with these general expressions, the examples below will be useful to understand how linear filtering performance and the theoretical bounds compare to eachother.

It is worthwhile to emphasize an interesting point in optimal linear filtering. The optimal filters $A(w)$ and $B(w)$ are bandpass filters, that is, only a certain portion of the channel bandwidth is required for transmission. Furthermore, the spectrum of the transmitted signal is non zero only for frequencies at which the signal power is larger than some threshold. This is very much consistent with the theoretical rate-distortion arguments where, in order to attain some distortion level, one needs to process only a certain part of the signal spectra for which the power is above some threshold. Another consequence of optimal linear filtering is that one only needs a bandlimited channel with an effective bandwidth $l_{\Omega}$ where $l_{\Omega}$ denotes the length of $\Omega=\left\{w: S(w)>\frac{1}{2 \nu}\right\}$.

\subsection{Examples}

We next study the linear filter design for important source statistics and compare the distortion attained by linear filtering to the theoretically attainable minimum distortion.

Example 1: Bandlimited message spectrum The power spectrum $S(w)>0$ for $w \leq W_{s}$ and zero otherwise. We first consider a message with a flat spectrum and then study a colored source with an arbitrary spectrum. We assume that the channel is an ideal bandlimited channel with $W_{\text {ch }} \geq W_{s}$. a) The source has a spectrum

$$
S(w)=\left\{\begin{array}{c}
\frac{\pi}{W_{s}},|w|<W_{s} \\
0, \text { else }
\end{array} .\right.
$$

In this case, it is easy to show that

$$
A(w)=\left\{\begin{array}{cc}
\sqrt{P}, & |w| \leq W_{s} \\
0, & \text { else }
\end{array}\right.
$$

and

$$
B(w)=\left\{\begin{array}{cc}
\frac{2 \pi \sqrt{P}}{2 \pi P+N_{0} W_{s}}, & |w| \leq W_{s} \\
0, & \text { else, }
\end{array},\right.
$$

that is, both filters become ideal lowpass filters whose pass-bands are the same as that of the message spectrum. The mean square error resulting from the optimal filters follows as $\xi_{i, o}=\frac{N_{0} W_{s}}{2 \pi P+N_{0} W_{s}}$. We note that this MSE is exactly the same as that would be attained by the theoretically optimum performance given by the rate-distortion function for a channel of bandwidth $2 W_{s}$ that is equal to the message bandwidth. Hence, we conclude that if a bandlimited signal with a flat spectrum is transmitted across an AWGN channel with the same bandwidth as the source, it sufficient to perform power scaling operation both at the transmitter and at the receiver to attain the minimum MSE, that is, linear processing is optimum in this case. On the other hand, if the bandwidth expansion is allowed, that is, the available transmission bandwidth is larger than the message bandwidth, optimum linear processing can not attain the theoretical minimum mean square error distortion given by $\xi_{R D}=\left(\frac{N_{0} W_{\mathrm{ch}}}{2 \pi P+N_{0} W_{\mathrm{ch}}}\right)^{\frac{W_{\mathrm{ch}}}{W_{s}}}$ [11].

b) Non-flat Spectrum: We now assume an arbitrary spectrum such that $S(w)>\epsilon$ for $w \leq W_{s}$ and zero otherwise and study the asymptotic performance of linear filtering as $\frac{P}{N_{0}} \rightarrow \infty$. Here, $\epsilon>0$ is some arbitrary real number and similar results follows in the limiting condition as $\epsilon \rightarrow 0$. For an asymptotic performance comparison, let us compute the distortion exponent $\mathcal{D}=\lim _{P / N_{0} \rightarrow \infty} \frac{\log \xi^{-1}\left(P / N_{0}\right)}{\log P / N_{0}}$ After some manipulations, we can show that the MSE at high SNR can be expressed as

$$
\xi=\frac{W_{s}}{\pi}\left[\frac{1}{W_{s}} \int_{0}^{W_{s}} \sqrt{S(w)} d w\right]^{2}\left[1+\frac{2 \pi P}{N_{0} W_{s}}\right]^{-1},
$$

thus, $\mathcal{D}=1$. Similarly, the theoretically optimum performance can be found by solving $R(D)=C$. Again, for high $P / N_{0}$, the theoretically minimum MSE can be obtained as

$$
D=\frac{W_{s}}{\pi} e^{\frac{1}{W_{s}} \int_{0}^{W_{s}} \log S(w) d w}\left[1+\frac{2 \pi P}{N_{0} W_{\mathrm{ch}}}\right]^{-\frac{W_{\mathrm{ch}}}{W_{s}}}
$$

Clearly, if $W_{\mathrm{ch}}=W_{s}$, the linear filtering attains the same distortion exponent as the optimal system does.

Example 2: Message with Butterworth Spectra We assume that

$$
S(w)=\frac{2 n \sin (\pi / 2 n)}{k\left(1+(w / k)^{2 n}\right)} .
$$

Note that $n=\infty$ corresponds to an ideal band-limited message spectrum like the one in Example 1. We also note that (15) is normalized such that $1 / 2 \pi \int S(w) d w=1$.

Substituting (15) into (4) and after some manipulations, we ob- 
tain

$$
\frac{\frac{2 \pi P}{k N_{0}}+\frac{w^{*}}{k}}{\sqrt{1+\left(w^{*} / k\right)^{2 n}}}=\frac{w^{*}}{k}{ }_{2} F_{1}\left(1 / 2 n, 1 / 2 ; 1+1 / 2 n,-\left(w^{*} / k\right)^{2 n}\right),
$$

where ${ }_{2} F_{1}(\cdot)$ is the Hypergeometric function. Solving for $w^{*}$ and substituting the result to (5) gives us the minimum MSE with optimal linear filtering:

$$
\begin{aligned}
\xi= & {\left[1-\operatorname{sinc}(1 / 2 n) w^{*} / k\left[{ }_{2} F_{1}\left(\frac{1}{2 n}, 1 ; 1+\frac{1}{2 n},-\left(w^{*} / k\right)^{2 n}\right)-\right.\right.} \\
& \left.\left.\frac{1}{\sqrt{1+\left(w^{*} / k\right)^{2 n}}}{ }_{2} F_{1}\left(\frac{1}{2 n}, 1 / 2 ; 1+\frac{1}{2 n},-\left(w^{*} / k\right)^{2 n}\right)\right]\right]
\end{aligned}
$$

The rate-distortion function for the source having Butterworth spectra can be evaluated as

$$
R(D)=\frac{n w_{o}}{\pi}\left[1-{ }_{2} F_{1}\left(1 / 2 n, 1 ; 1+1 / 2 n,-\left(w_{o} / k\right)^{2 n}\right)\right]
$$

where $w_{o}$ is obtained from

$$
D=\left[1-\operatorname{sinc}\left(\frac{1}{2 n}\right) \frac{w_{O}}{k}\left[{ }_{2} F_{1}\left(\frac{1}{2 n}, 1 ; 1+\frac{1}{2 n},-\left(\frac{w_{O}}{k}\right)^{2 n}\right)-\frac{1}{1+\left(\frac{w_{O}}{k}\right)^{2 n}}\right]\right]
$$

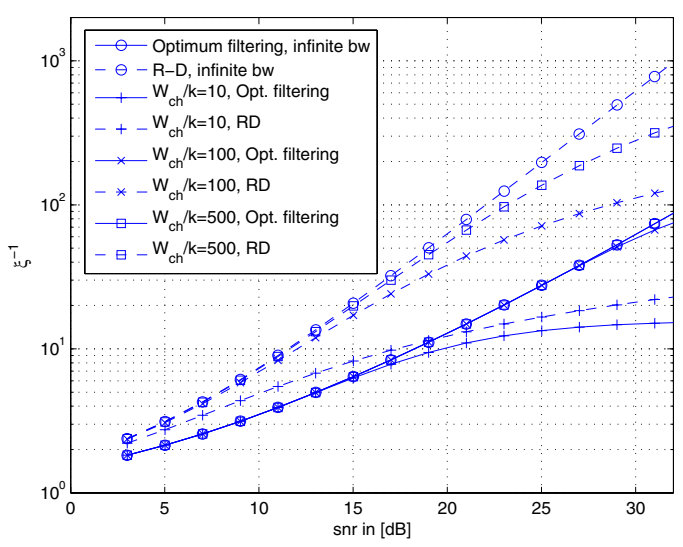

Fig. 4. MSE for the message with a single-pole Butterworth spectrum.

The resulting expressions are evaluated numerically for various channel models and the resulting MSE curves are plotted in Figure 4 when $n=1$ (a single-pole Butterworth spectrum). The plots indicate that for this message spectrum, if the bandwidth of the channel is high, e.g., $W_{\mathrm{ch}} / k=100$ or 500 , optimal RD performance is significantly better than the linear filtering performance. On the other hand, for transmission over low bandwith channels, linear filtering performs close to the rate-distortion performance; for instance, we are about only $2.2 \mathrm{~dB}$ away from the rate-distortion performance at an MSE of 0.1 if $W_{\mathrm{ch}}=10 k$, and the difference gets less and less as bandwidth is decreased. This result is promising for sensor networks where we have hardlimits on power and bandwidth since in such cases, using liner processing (with the optimum filters) may be sufficient to attain an acceptable estimation quality.

\section{CONCLUSIONS}

We studied the communication problem in the presence of a power constraint on the transmitted signal. We restricted our attention to the class of linear processing and derived the optimum transmit and receive filters that minimize the mean square error subject to a transmit power constraint. For the transmission of stationary signals over linear time-invariant channels, explicit expressions for the frequency responses of the filters and the mean square error that can be attained with these filters are derived. The main result of these derivations is that the optimum transmit and receive filters are bandlimited filters which pass certain frequency components of the signal at which the spectral density is above some threshold. This result is agreeing with the optimum source coding criterion which says that it is sufficient to represent the signal with its high-power frequency components and ignore those frequency components at which the power spectrum is small. Numerical examples indicate that the optimum linear filtering attains less mean square error performance than those attained by the optimum Wiener filtering where that transmit filter is simply an amplify-and forward filter. The results can be readily extended for the discrete time-systems. The journal version of this paper will also include more general cases including the transmission of non-stationary and quasi-stationary sources, the optimality of linear FIR filters for discrete-time signal transmission subject to power constraints.

\section{REFERENCES}

[1] T. J. Goblick, "Theoretical limitations on the transmission of data from analog sources," IEEE Transactions on Information Theory, vol. 11, no. 4, pp. 558-567, Oct 1965.

[2] R. G. Gallager, Information theory and realiable communication, John Wiley and Sons, New York, NY, 1968.

[3] T. Berger, Rate Distortion Theory, Prentice-Hall, Englewood Cliffs, N.J., 1971.

[4] J. P. Costas, "Coding with linear systems," Proc. IRE, vol. 40, pp. 1101-1103, Sept 1952.

[5] D. W. Tufts, "Nyquists problem-the joint optimization of transmitter and receiver in pulse amplitude modulation," Proc. IEEE, vol. 53, pp. 248-259, 1965.

[6] T. Berger and D. W. Tufts, "Optimum pulse amplitude modulation, part i: transmitter-receiver design and bounds from information theory," IEEE Transactions on Information Theory, vol. 13, pp. 196-208, Apr 1967.

[7] D. Chan and R. W. Donaldson, "Optimum pre- and postfiltering of sampled signals with application to pulse modulation and data. compression systems," IEEE Trans. Communication Technology, vol. 19, no. 2, pp. 141-157, April 1971.

[8] K.-H. Lee and D. P. Petersen, "Optimal linear precoding for vector channels," IEEE Trans. Commun., vol. 24, no. 12, pp. 1283-1290, Dec 1976.

[9] T. Basar and B. Sankur, "Performance bounds on optimal linear coding for discrete-time multichannel communications systems," IEEE Trans. Inform. Thoery, vol. 26, no. 2, pp. 212217, Mar 1980.

[10] H. S. Malvar and D. H. Staelin, "Optimal pre- and postfilters for multichannel signal processing," IEEE Trans. Acoustics, Speech and Signal Processing, vol. 36, no. 2, pp. 287-289, Feb 1988.

[11] H. L. Van Trees, Detection, Estimation and Modulation Theory, Part II: Nonlinear Modulation Theory, John Wiley and Sons, Inc., New York, 1971.

[12] N. Wiener, Extrapolation, Interpolation, and Smoothing of Stationary Time Series, MIT Press, Cambridge, MA, 1949.

[13] R. C. Booton Jr., "An optimization theory for time-varying linear systems with nonstationary statistical inputs," Proceedings of the I.R.E., vol. 40, pp. 977-981, 1952. 